Western University

Scholarship@Western

$7-1-2020$

\title{
Screening and Treatment Outcomes in Adults and Children With Type 1 Diabetes and Asymptomatic Celiac Disease: The CD-DIET Study.
}

\author{
Farid H Mahmud \\ Antoine B M Clarke \\ Kariym C Joachim \\ Esther Assor \\ Charlotte McDonald
}

See next page for additional authors

Follow this and additional works at: https://ir.lib.uwo.ca/paedpub

Part of the Pediatrics Commons

\section{Citation of this paper:}

Mahmud, Farid H; Clarke, Antoine B M; Joachim, Kariym C; Assor, Esther; McDonald, Charlotte; Saibil, Fred; Lochnan, Heather A; Punthakee, Zubin; Parikh, Amish; Advani, Andrew; Shah, Baiju R; Perkins, Bruce A; Zuijdwijk, Caroline S; Mack, David R; Koltin, Dror; De Melo, Emilia N; Hsieh, Eugene; Mukerji, Geetha; Gilbert, Jeremy; Bax, Kevin; Lawson, Margaret L; Cino, Maria; Beaton, Melanie D; Saloojee, Navaaz A; Lou, Olivia; Gallego, Patricia H; Bercik, Premysl; Houlden, Robyn L; Aronson, Ronnie; Kirsch, Susan E; Paterson, William G; and Marcon, Margaret A, "Screening and Treatment Outcomes in Adults and Children With Type 1 Diabetes and Asymptomatic Celiac Disease: The CD-DIET Study." (2020). Paediatrics Publications. 540. https://ir.lib.uwo.ca/paedpub/540 


\section{Authors}

Farid H Mahmud, Antoine B M Clarke, Kariym C Joachim, Esther Assor, Charlotte McDonald, Fred Saibil, Heather A Lochnan, Zubin Punthakee, Amish Parikh, Andrew Advani, Baiju R Shah, Bruce A Perkins, Caroline S Zuijdwijk, David R Mack, Dror Koltin, Emilia N De Melo, Eugene Hsieh, Geetha Mukerji, Jeremy Gilbert, Kevin Bax, Margaret L Lawson, Maria Cino, Melanie D Beaton, Navaaz A Saloojee, Olivia Lou, Patricia H Gallego, Premysl Bercik, Robyn L Houlden, Ronnie Aronson, Susan E Kirsch, William G Paterson, and Margaret A Marcon 


\section{Screening and Treatment Outcomes in Adults and Children With Type 1 Diabetes and Asymptomatic Celiac Disease: The CD-DIET Study}

https://doi.org/10.2337/dc19-1944

\section{OBJECTIVE}

To describe celiac disease (CD) screening rates and glycemic outcomes of a gluten-free diet (GFD) in patients with type 1 diabetes who are asymptomatic for CD.

\section{RESEARCH DESIGN AND METHODS}

Asymptomatic patients (8-45 years) were screened for CD. Biopsy-confirmed CD participants were randomized to GFD or gluten-containing diet (GCD) to assess changes in $\mathrm{HbA}_{1 \mathrm{c}}$ and continuous glucose monitoring over 12 months.

\section{RESULTS}

Adults had higher CD-seropositivity rates than children $(6.8 \%$ [95\% $\mathrm{Cl}$ 4.9-8.2\%, $N=$ $1,298]$ vs. $4.7 \%[95 \% \mathrm{Cl} 3.4-5.9 \%, N=1,089], P=0.035)$ with lower rates of prior $\mathrm{CD}$ screening ( $6.9 \%$ vs. $44.2 \%, P<0.0001)$. Fifty-one participants were randomized to a GFD $(N=27)$ or GCD $(N=24)$. No $\mathrm{HbA}_{1 \mathrm{c}}$ differences were seen between the groups $(+0.14 \%, 1.5 \mathrm{mmol} / \mathrm{mol} ; 95 \% \mathrm{Cl}-0.79$ to $1.08 ; P=0.76)$, although greater postprandial glucose increases $(4-\mathrm{h}+1.5 \mathrm{mmol} / \mathrm{L} ; 95 \% \mathrm{Cl} 0.4-2.7 ; P=0.014)$ emerged with a GFD.

\section{CONCLUSIONS}

$C D$ is frequently observed in asymptomatic patients with type 1 diabetes, and clinical vigilance is warranted with initiation of a GFD.

Celiac disease (CD) is an immune reaction-mediated condition triggered by gluten, a protein found in wheat, barley, and rye. CD risk is increased in patients with type 1 diabetes and is characterized by a broad spectrum of presentations, including gastrointestinal symptoms, growth alterations and anemia, but many patients are asymptomatic (1-4).

Serologic screening for $C D$ has high sensitivity and specificity, but few reports have evaluated $C D$ rates and the impact of treatment with a gluten-free diet (GFD) in asymptomatic $\mathrm{CD}(\mathrm{aCD})$ patients with type 1 diabetes. The Celiac Disease and Diabetes-Dietary Intervention and Evaluation Trial (CD-DIET) prospectively screened a large cohort of patients aged $8-45$ years with subsequent engagement in a randomized, dietary treatment trial to evaluate changes in $\mathrm{HbA}_{1 \mathrm{c}}$ and glycemic variability.
Farid H. Mahmud, ${ }^{1}$ Antoine B.M. Clarke, ${ }^{1}$ Kariym C. Joachim, ${ }^{1}$ Esther Assor, ${ }^{1}$ Charlotte McDonald, ${ }^{2}$ Fred Saibil, ${ }^{3}$ Heather A. Lochnan, ${ }^{4}$ Zubin Punthakee, ${ }^{5}$ Amish Parikh, ${ }^{6}$ Andrew Advani, ${ }^{7}$ Baiju R. Shah, ${ }^{8}$ Bruce A. Perkins, ${ }^{9}$ Caroline S. Zuijdwijk, ${ }^{10}$ David R. Mack, ${ }^{11}$ Dror Koltin, ${ }^{6}$ Emilia N. De Melo, ${ }^{1}$ Eugene Hsieh, ${ }^{12}$ Geetha Mukerii, ${ }^{13}$ Jeremy Gilbert, ${ }^{8}$ Kevin Bax, ${ }^{14}$ Margaret L. Lawson, ${ }^{10}$ Maria Cino, ${ }^{15}$ Melanie D. Beaton, ${ }^{16}$ Navaaz A. Saloojee ${ }^{17}$ Olivia Lou, ${ }^{18}$ Patricia H. Gallego, ${ }^{19}$ Premysl Bercik, ${ }^{20}$ Robyn L. Houlden, ${ }^{21}$ Ronnie Aronson, ${ }^{22}$ Susan E. Kirsch, ${ }^{23}$ William G. Paterson, ${ }^{24}$ and Margaret A. Marcon $^{25}$

${ }^{1}$ Division of Endocrinology, Department of Pediatrics, The Hospital for Sick Children, University of Toronto, Toronto, Ontario, Canada

${ }^{2}$ Division of Endocrinology and Metabolism, St. Joseph's Health Care, London Health Sciences Centre, Western University, London, Ontario, Canada

${ }^{3}$ Division of Gastroenterology, Sunnybrook Health Sciences Centre, University of Toronto, Toronto, Ontario, Canada

${ }^{4}$ Department of Endocrinology, The Ottawa Hospital, Ottawa, Ontario, Canada

${ }^{5}$ Department of Medicine, McMaster University, Hamilton, Ontario, Canada

${ }^{6}$ Division of Endocrinology, Trillium Health Partners, Toronto, Ontario, Canada

${ }^{7}$ Division of Endocrinology, St. Michael's Hospital, Toronto, Ontario, Canada

${ }^{8}$ Division of Endocrinology and Metabolism, Sunnybrook Health Sciences Centre, Toronto, Ontario, Canada

${ }^{9}$ Division of Endocrinology and Metabolism, University Health Network, University of Toronto, Toronto, Ontario, Canada

${ }^{10}$ Division of Endocrinology and Metabolism, University of Ottawa, Children's Hospital of Eastern Ontario, Ottawa, Ontario, Canada

${ }^{11}$ Division of Gastroenterology, Hepatology and Nutrition, University of Ottawa, Children's Hospital of Eastern Ontario, Ottawa, Ontario, Canada

${ }^{12}$ Department of Pathology, Sunnybrook Health Sciences Centre, Toronto, Ontario, Canada

${ }^{13}$ Division of Endocrinology, Women's College Hospital, Toronto, Ontario, Canada

${ }^{14}$ Pediatric Gastroenterology, Department of Pediatrics, Children's Hospital, London Health Sciences Centre, Western University, London, Ontario, Canada

${ }^{15}$ Division of Gastroenterology, University Health Network, University of Toronto, Toronto, Ontario, Canada

${ }^{16}$ Division of Gastroenterology, London Health Sciences Centre, London, Ontario, Canada 


\section{RESEARCH DESIGN AND METHODS}

\section{Recruitment of Participants}

Patients aged 8-45 years with type 1 diabetes ( $\geq 1$-year duration) were approached to undergo $C D$ testing at $22 \mathrm{di}-$ abetes centers in Ontario, Canada as part of the CD-DIET Study (clinicaltrials.gov, NCT01566110) (5). Exclusion criteria included CD symptoms (gastrointestinal symptomology or evidence of growth impairment, anemia, or osteoporosis), a previous CD diagnosis, and pregnancy.

\section{Screening}

Eligible participants (Supplementary Fig. 1) were screened using tissue transglutaminase IgA (TTG-IgA; Inova Diagnostics). CD-seropositive (TTG-IgA $\geq 30$ chemiluminescent units [CU]) participants progressed to a gastroenterology consultation with centralized biopsy confirmation (Marsh score $\geq 2$ ).

\section{Dietary Intervention Study}

Interested participants with biopsy-confirmed $C D$ were randomized in a 1:1 ratio to a GFD or a gluten-containing diet (GCD) and followed for 1 year. Study visits with a dietitian occurred every 3 months using a standardized educational curriculum. Dietary adherence was also evaluated through quantification of dietary gluten intake and repeat TTG-IgA testing. Safety laboratory tests were evaluated (see Supplementary Appendix) with monitoring of growth, symptomology, and pregnancy. GCD participants who became symptomatic were transitioned to a GFD with the completion of outcome measures. A Data Safety Monitoring Committee evaluated adverse events. The primary outcome of CD-DIET was the change in $\mathrm{HbA}_{1 \mathrm{c}}$ from baseline to 12 months. $\mathrm{HbA}_{1 \mathrm{c}}$ was assessed centrally at baseline and at 6 and 12 months, with additional testing by local laboratories. Continuous glucose monitoring (CGM)
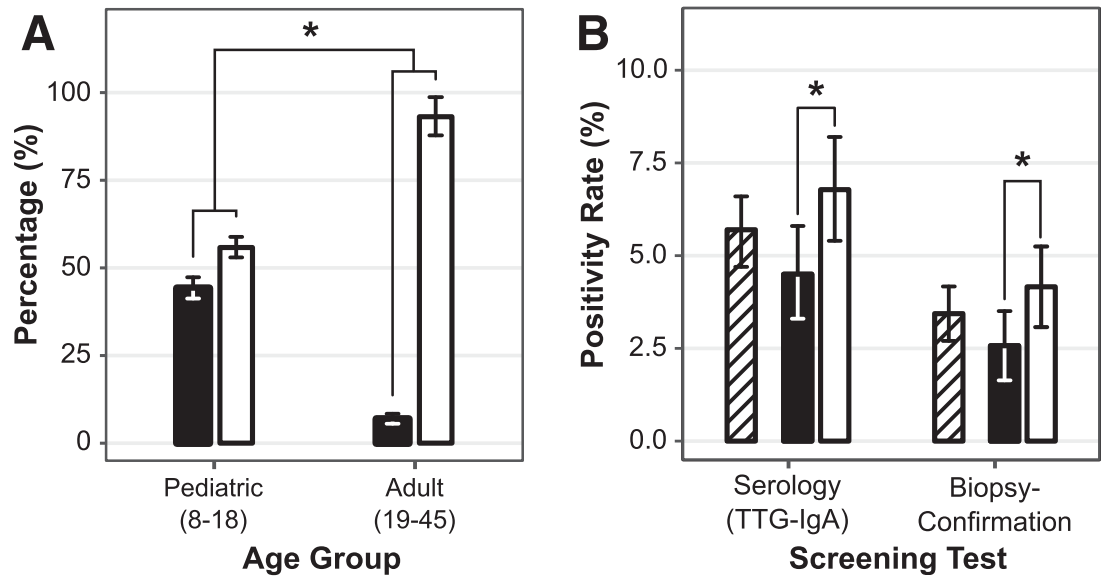

Screening Test

Figure 1-The results from the CD-DIET screening of asymptomatic patients with type 1 diabetes. $A$ : The percentage of participants previously (black bars) and nonpreviously (white bars) screened for $C D$ by age group showing lower screening rates in adults $(6.9 \%$ vs. $44.2 \%, P<0.0001)$. $B$ : $C D$ serology- (TTG-IgA) and CD biopsy-confirmed rates of $C D$ among the 2,387 subjects screened. Hatched bars indicate combined rates of $C D$ serology and biopsy positivity, black bars illustrate pediatric data ( $8-18$ years, $N=1,089$ ), while white bars show adult rates $(19-44$ years, $N=1,298$ ). Error bars represent $95 \%$ Cls. ${ }^{*}$ Significant differences below $P<0.05$.

was conducted using the iPro2 CGM system (Medtronic Inc.) for blinded assessment.

Analysis was by intention to treat. Longitudinal differences in $\mathrm{HbA}_{1 \mathrm{c}}$ between groups were analyzed using the difference in differences approach and confirmed using linear mixed effects regression. CGM results were summarized using standard parametric methods, while paired $t$ tests were used to assess within-group differences in $\mathrm{HbA}_{1 \mathrm{c}}$ and postprandial glucose levels. All analyses were completed using R Statistics 3.5.1.

A sample size of $n=60$ (30 patients per arm) was determined to be sufficient for our between-group analysis to reach $80 \%$ power at a $5 \%$ significance level under two-sided conditions. Paired analysis of $\mathrm{HbA}_{1 \mathrm{c}}$ in the GFD arm, a sample size of $n=25$ before versus after pairs reached a power above $80 \%$ using a two-sided paired $t$ test with significance set at $P=0.05$.

\section{RESULTS}

Recruitment and CD Screening Overall, 2,585 patients consented and 2,387 subjects completed CD screening, including 1,298 adults $(54.4 \%, 19-45$ years) and 1,089 children $(45.6 \%, 8-18$ years) (see Supplementary Appendix). Higher rates of any prior CD serologic screening were seen in pediatric participants ( $n=475,43.6 \%, 95 \% \mathrm{Cl} 40.7-46.5 \%)$ as compared with adult participants ( $n=89,6.9 \%, 95 \% \mathrm{Cl} 5.5-8.3 \%$ ) (odds ratio [OR] 10.5, $P<0.0001$ ) (Fig. 1A). Adults had higher CD-seropositivity rates $(n=88,6.8 \%$; $95 \% \mathrm{Cl} 5.4-8.2 \%)$ compared with children $(n=51 ; 4.7 \%$; $\mathrm{OR}=1.48,95 \% \mathrm{Cl} 3.4-5.9 \%, P=0.035)$, but rates did not differ within each age group on the basis of previous CD screening status. Biopsies were completed in 104 participants, and 82 were confirmed by biopsy with CD-biopsypositivity rates of $4.2 \%(n=54 ; 95 \%$ $\mathrm{Cl} 3.1-5.3 \%)$ and $2.6 \%(n=28 ; 95 \%$

\footnotetext{
${ }^{17}$ Department of Gastroenterology, The Ottawa Hospital, Ottawa, Ontario, Canada

${ }^{18}$ JDRF, New York, NY

${ }^{19}$ Division of Endocrinology, Children's Hospital, London Health Sciences Centre, Western University, London, Ontario, Canada

${ }^{20}$ Department of Gastroenterology, McMaster University, Hamilton, Ontario, Canada

${ }^{21}$ Department of Endocrinology, Kingston General Hospital, Kingston, Ontario, Canada

${ }^{22}$ LMC Diabetes \& Endocrinology, Toronto, Ontario, Canada
}

${ }^{23}$ Division of Endocrinology, Markham-Stouffville Hospital, Markham, Ontario, Canada

${ }^{24}$ Department of Gastroenterology, Kingston General Hospital, Kingston, Ontario, Canada

${ }^{25}$ Division of Gastroenterology, Hepatology and Nutrition, Department of Pediatrics, The Hospital for Sick Children, University of Toronto, Toronto, Ontario, Canada

Corresponding author: Farid H. Mahmud, farid. mahmud@sickkids.ca

Received 30 September 2019 and accepted 25 March 2020
Clinical trial reg. no. NCT01566110, clinicaltrials gov

This article contains supplementary material online at Figshare: https://doi.org/10.2337/ figshare.12041016.

(C) 2020 by the American Diabetes Association Readers may use this article as long as the work is properly cited, the use is educational and not for profit, and the work is not altered. More information is available at https://www.diabetesjournals .org/content/license. 
$\mathrm{Cl} 1.6-3.5 \%)$ for adult and pediatric participants (OR $=1.64, P=0.042$ ) (Fig. $1 B$ ).

\section{Dietary Intervention Study}

The 51 participants were randomized into the intervention phase, with 27 to the GFD group and 24 to the GCD group. Adherence to the assigned diet, as assessed by serial measures of gluten intake and TTG antibody titers, was deemed excellent (Fig. 2A). Ultimately, we observed no longitudinal difference in $\mathrm{HbA}_{1 \mathrm{c}}$ between the GFD group and GCD group $(+0.14 \%$ [1.5 $\mathrm{mmol} / \mathrm{mol}], 95 \% \mathrm{Cl}-0.79$ to $1.08, P=$ $0.76)$. Within the GFD group, an increase in $\mathrm{HbA}_{1 \mathrm{c}}$ of $+0.30 \%$ (3.3 $\mathrm{mmol} / \mathrm{mol}$ ) was observed over 12 months $(95 \% \mathrm{Cl} 0.04-$ $0.57 \%, P=0.028$ ) (Fig. $2 B$ ).

Using blinded CGM, no differences were seen in the percentage of time spent in hypoglycemic, euglycemic, or hyperglycemic ranges at 12 months. Despite this, postprandial glycemia was higher with the GFD relative to premeal levels at both the 2-h ( $+1.6 \mathrm{mmol} / \mathrm{L}, 95 \% \mathrm{Cl} 0.7-2.6$, $P=0.0015)$ and 4-h (+1.5 mmol/L, 95\% Cl 0.4-2.7, $P=0.014$ ) time points, while glucose levels of the GCD arm returned to premeal levels ( $\Delta=0.1 \mathrm{mmol} / \mathrm{L}$ after $4 \mathrm{~h}$; $95 \% \mathrm{Cl}-0.8$ to 1.0 ) (Fig. 2 C). No differences in growth parameters (including height, weight, or BMI in children and adults, Supplementary Figs. 2 and 3) nor total daily insulin dose or total daily insulin dose per $\mathrm{kg}$ were observed between groups.

Hypoglycemic adverse events that were nonsevere were reported with the GCD group $(N=3,12.5 \%)$, with none reported with the GFD group ( $P=0.097)$.

\section{CONCLUSIONS}

This study describes screening and treatment outcomes from a large prospective assessment of adult and pediatric patients with type 1 diabetes with aCD. We observed high rates of $\mathrm{aCD}$ with 1.5 -fold higher rates in adults as compared with children. This is consistent with increasing rates of CD in adults (6) and changes in the symptom profile toward a more subtle and less classical malabsorptive presentation with increasing age (7). In addition, age-related increases in autoimmune comorbidities are also well recognized, with recent T1D Exchange clinic registry data confirming rising rates of autoimmune comorbidities from adolescence into adulthood $(8,9)$.

Adult participants in our study were also less likely to be screened for CD than children. This may reflect a lack of clarity about CD screening recommendations in adults and the absence of outcome studies in this population. Current North American diabetes guidelines advise consideration of screening patients with type 1 diabetes for $C D$, but actual details regarding screening tests, timing, and intervals are only explicitly described for children $(10,11)$.

Dietary intervention with a GFD did not result in a difference in $\mathrm{HbA}_{1 \mathrm{c}}$ between randomized groups but led to greater postprandial glucose excursions relative to a GCD. These changes are consistent with mucosal recovery, which can be prolonged (1-3 years), with subsequent improvements in absorption $(2,12)$. Nonetheless, transition to a GFD was safe with no significant differences observed in hypoglycemic time or adverse events.

Strengths of this report include the large number of patients screened and the rigor in delineating an asymptomatic cohort. In addition, participants in the intervention phase, who were provided with frequent dietitian-based support, exhibited clear differences in gluten intake and follow-up TTG serology. Limitations of this study include a small sample size and the heterogeneity of participant characteristics that limits generalization of these findings. In addition, our data do not represent the prevalence rates, as we were challenged by enumeration of the complete population at risk in the clinics where screening was completed. It was also our experience that despite facilitated and no-cost access to care, many asymptomatic participants were overwhelmed by the diagnostic process and by the prospect of managing these two conditions, each with significant lifestyle implications.

In summary, diabetes clinicians should be aware of $C D$ as an important autoimmune comorbidity of type 1 diabetes that is frequently asymptomatic. In addition, clinical vigilance is warranted during dietary transition, as GFD-treatment may impact short-term glycemic variability.
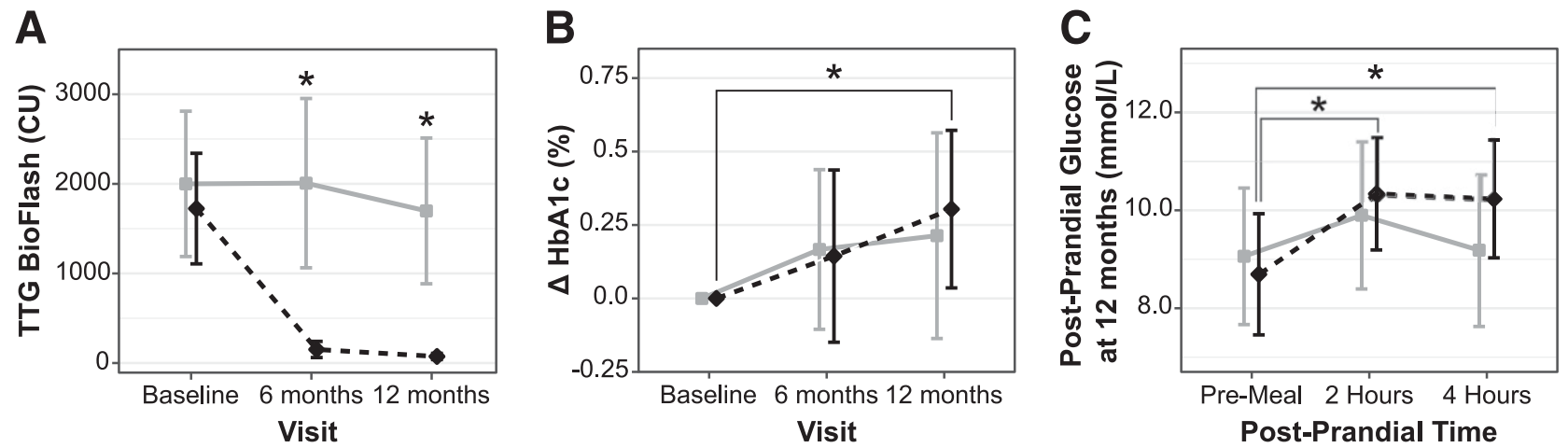

Figure 2-The results from the CD-DIET Dietary Intervention Trial. Data from the GFD arm shown in black with a dashed line ( $\square$ ), while data from the GCD are shown with solid gray line (घ). A: Dietary adherence to assigned group was assessed using serial serologic testing. TTG-IgA antibody titers were similar in the GCD and the GFD groups at baseline. They appropriately decreased in the GFD arm by 6 months and continued to drop among GFDs, resulting in a significant difference between GCDs $(1,698.0 \pm 1,834.0 \mathrm{CU})$ and GFDs $(73.4 \pm 83.9 \mathrm{CU})$ at 12 months $(P<0.0001)$. $B$ : The change in $\mathrm{HbA} \mathrm{A}_{1 \mathrm{c}}$ between study groups. No difference in $\mathrm{HbA}_{1 \mathrm{c}}$ was seen between the the $\mathrm{GFD}$ group relative those on a GCD over the $12 \mathrm{months}(+0.14 \%$ [ $1.5 \mathrm{mmol} / \mathrm{mol}$ ], $P=$ $0.76)$; however, within the GFD group, $\mathrm{HbA}_{1 \mathrm{c}}$ increased $(+0.30 \%[3.3 \mathrm{mmol} / \mathrm{mol}], 95 \% \mathrm{Cl} 0.04-0.57 \%, P=0.028)$. $C$ : Changes in postprandial glucose assessed by CGM. Postprandial glycemia in the GFD was higher compared with premeal levels at both the $2-\mathrm{h}$ time point $(+1.6 \mathrm{mmol} / \mathrm{L}[29 \mathrm{mg} / \mathrm{dL}] ; P=$ $0.0015)$ and 4 -h time point $(+1.5 \mathrm{mmol} / \mathrm{L}[27 \mathrm{mg} / \mathrm{dL}] ; P=0.014)$, while glucose levels of the GCD arm returned to premeal levels $(\Delta=0.1 \mathrm{mmol} / \mathrm{L}$ $[1.8 \mathrm{mg} / \mathrm{dL}])$. Error bars represent $95 \%$ Cls. ${ }^{*}$ Significant differences below $P<0.05$. 
Given the additional risks for CD-related microvascular and bone complications, longitudinal studies are also needed to assess the clinical impact of this double diagnosis across the patient's life span (13-15).

Acknowledgments. This trial was funded by JDRF Canadian Clinical Trial Network. We are grateful to the research staff at clinical sites and to patients and their families who participated in this trial.

Duality of Interest. F.H.M. served on advisory boards for Eli Lilly and Insulet. E.A. was employed as a dietician with CD-DIET. Z.P. received grants, speaker fees, and consulting fees from Abbott, AstraZeneca, Boehringer Ingelheim, Bristol-Myers Squibb, Eli Lilly, Janssen, Lexicon, Merck, Medtronic, Novo Nordisk, Pfizer, and Sanofi. A.P. received consulting fees for education-related activities and advisory boards from Medtronic, Sanofi, Novo Nordisk, Janssen, AstraZeneca, Boehringer Ingelheim, Lilly. A.A. received research support from Boehringer Ingelheim and AstraZeneca, is listed as an inventor on a patent application (WO 2015/128453) from Boehringer Ingelheim, has received an unrestricted educational grant from Eli Lilly, and has received honoraria from Novo Nordisk, Eli Lilly, Boehringer Ingelheim, Abbott, and Dexcom. B.A.P. received personal fees from Medtronic, Lilly, Abbott, Novo Nordisk, Insulet, Sanofi, Janssen, and Boehringer Ingelheim, as well as grants from the Bank of Montreal and Boehringer Ingelheim, all outside the scope of the submitted work. C.S.Z. is currently a coinvestigator for a Children's Hospital of Eastern Ontario site of the Medtronic multicentre randomized controlled trial of home use of a hybrid closed-loop system (no monetary gain) and participated in a Lilly Canada Advisory board once in February 2019 (honorarium directed to institution). J.G. received honoraria for speaking from Abbott, AstraZeneca, Boerhinger Ingelheim, Dexcom, Eli Lilly, Insulet, Janssen, Merck, Novo Nordisk, and Sanofi and is on advisory boards for AstraZeneca, Boerhinger, Dexcom Eli Lilly, Janssen, Merck, Novo Nordisk, and Sanofi. M.D.B. received honoraria from Shire and Allergan, served on advisory boards for AbbVie, Takeda, Janssen, Lupin, and Gilead, and received grants from AbbVie, Novo Nordisk, and Gilead. R.L.H. participated in clinical trials (in the last 2 years) for AstraZeneca, Boehringer Ingelheim, Eli Lilly, and Novo Nordisk. R.A. received research grants from Sanofi, Novo Nordisk,
Janssen, AstraZeneca, Becton Dickinson Technologies, Boehringer Ingelheim, Eli Lilly, Zealand, Xeris, Medpace, Kowa, Insulet, Dexcom, Bausch Health, Tandem Diabetes, and Bayer and receives consultancies and personal fees from Sanofi, Novo Nordisk, Janssen, AstraZeneca, Becton Dickinson Technologies, Boehringer Ingelheim, Eli Lilly, HTL Strefa, Gilead, and Merck. No other potential conflicts of interest relevant to this article were reported.

Author Contributions. F.H.M., A.B.M.C., E.A. and M.A.M. wrote the first draft of the report and contributed to the study design, statistical analysis, and data interpretation. A.B.M.C., K.C.J., C.M., F.S., H.A.L., Z.P., A.P., A.A., B.R.S., B.A.P., C.S.Z., D.R.M., D.K., E.H., G.M., J.G., K.B., M.L.L., M.C., M.D.B., N.A.S., O.L., P.H.G., P.B., R.L.H., R.A., S.E.K., and W.G.P. were involved in the data collection and analyses. F.H.M., K.C.J., E.A., C.M., H.A.L., Z.P., A.P., A.A., B.R.S., B.A.P., C.S.Z., D.K., G.M., J.G., M.L.L., P.H.G., R.L.H., R.A., S.E.K., and M.A.M. recruited patients into the study. K.C.J., C.M., F.S., H.A.L., Z.P., A.P., A.A., B.R.S., B.A.P., C.S.Z., D.R.M., D.K., E.N.D.M., E.H., G.M., J.G., K.B., M.L.L., M.C., M.D.B., N.A.S., O.L., P.H.G., P.B., R.L.H., R.A., S.E.K., and W.G.P. contributed to the drafting and review of the report. F.H.M. is the guarantor of this work and, as such, had full access to all the data in the study and takes responsibility for the integrity of the data and the accuracy of the data analysis.

Prior Presentation. Parts of this study were presented at the 2019 European Association for the Study of Diabetes Conference (EASD 2019), 16-20 September 2019, Barcelona, Spain.

\section{References}

1. Lebwohl B, Sanders DS, Green PHR. Coeliac disease. Lancet 2018;391:70-81

2. Rubio-Tapia A, Hill ID, Kelly CP, Calderwood AH, Murray JA; American College of Gastroenterology. ACG clinical guidelines: diagnosis and management of celiac disease. Am J Gastroenterol 2013;108:656-676; quiz 677

3. Elfström P, Sundström J, Ludvigsson JF. Systematic review with meta-analysis: associations between coeliac disease and type 1 diabetes. Aliment Pharmacol Ther 2014;40:1123-1132

4. Mahmud FH, Elbarbary NS, Fröhlich-Reiterer E, et al. ISPAD Clinical Practice Consensus Guidelines 2018: other complications and associated conditions in children and adolescents with type 1 diabetes. Pediatr Diabetes 2018;19(Suppl. 27):275-286

5. Mahmud FH, De Melo EN, Noordin K, et al. The Celiac Disease and Diabetes-Dietary Intervention and Evaluation Trial (CD-DIET) protocol: a randomised controlled study to evaluate treatment of asymptomatic coeliac disease in type 1 diabetes. BMJ Open 2015;5:e008097

6. Kang JY, Kang AH, Green A, Gwee KA, Ho KY. Systematic review: worldwide variation in the frequency of coeliac disease and changes over time. Aliment Pharmacol Ther 2013;38:226-245 7. Volta U, Caio G, Stanghellini V, De Giorgio R. The changing clinical profile of celiac disease: a 15-year experience (1998-2012) in an Italian referral center. BMC Gastroenterol 2014;14:194 8. Hughes JW, Riddlesworth TD, DiMeglio LA, Miller KM, Rickels MR, McGill JB; T1D Exchange Clinic Network. Autoimmune diseases in children and adults with type 1 diabetes from the T1D Exchange Clinic Registry. J Clin Endocrinol Metab 2016;101:4931-4937

9. Barker JM, Yu J, Yu L, et al. Autoantibody "subspecificity" in type 1 diabetes: risk for organspecific autoimmunity clusters in distinct groups. Diabetes Care 2005;28:850-855

10. American Diabetes Association. 4. Comprehensive medical evaluation and assessment of comorbidities: Standards of Medical Care in Diabetes-2020. Diabetes Care 2020;43(Suppl. 1):S37-S47

11. Wherrett DK, Ho J, Huot C, Legault L, Nakhla M, Rosolowsky E; Diabetes Canada Clinical Practice Guidelines Expert Committee. Type 1 diabetes in children and adolescents. Can J Diabetes 2018;42(Suppl. 1):S234-S246

12. Galli G, Esposito G, Lahner E, et al. Histological recovery and gluten-free diet adherence: a prospective 1-year follow-up study of adult patients with coeliac disease. Aliment Pharmacol Ther 2014;40:639-647

13. Kamycheva E, Goto T, Camargo CA, Jr. Celiac disease is associated with reduced bone mineral density and increased FRAX scores in the US National Health and Nutrition Examination Survey. Osteoporos Int 2017;28:781-790

14. Rohrer TR, Wolf J, Liptay S, et al.; DPV Initiative and the German BMBF Competence Network Diabetes Mellitus. Microvascular complications in childhood-onset type 1 diabetes and celiac disease: a multicenter longitudinal analysis of 56,514 patients from the GermanAustrian DPV Database. Diabetes Care 2015;38: 801-807

15. Mollazadegan K, Kugelberg M, Montgomery SM, Sanders DS, Ludvigsson J, Ludvigsson JF. A population-based study of the risk of diabetic retinopathy in patients with type 1 diabetes and celiac disease. Diabetes Care 2013;36:316-321 